\title{
Rates of voluntary and compulsory psychiatric in-patient treatment in England: an ecological study investigating associations with deprivation and demographics ${ }^{\dagger}$
}

Patrick Keown, Orla McBride, Liz Twigg, David Crepaz-Keay, Eva Cyhlarova, Helen Parsons, Jan Scott, Kamaldeep Bhui and Scott Weich

\section{Background}

Individual variables and area-level variables have been identified as explaining much of the variance in rates of compulsory in-patient treatment.

\section{Aims \\ To describe rates of voluntary and compulsory psychiatric in-patient treatment in rural and urban settings in England, and to explore the associations with age, ethnicity and deprivation.}

\section{Method}

Secondary analysis of 2010/11 data from the Mental Health Minimum Dataset.

\section{Results}

Areas with higher levels of deprivation had increased rates of in-patient treatment. Areas with high proportions of adults aged 20-39 years had the highest rates of compulsory in-patient treatment as well as the lowest rates of voluntary in-patient treatment. Urban settings had higher rates of compulsory in-patient treatment and ethnic density was associated with compulsory treatment in these areas. After adjusting for age, deprivation and urban/rural setting, the association between ethnicity and compulsory treatment was not statistically significant.

\section{Conclusions}

Age structure of the adult population and ethnic density along with higher levels of deprivation can account for the markedly higher rates of compulsory in-patient treatment in urban areas.

\section{Declaration of interest}

None.

\section{Copyright and usage}

(c) The Royal College of Psychiatrists 2016.
Mental health services in England have been transformed over the past three decades through the transfer of most care from hospital to the community, where services are available 24 hours a day. ${ }^{1-4}$ It is therefore surprising that detentions under the Mental Health Act have risen steadily over the same time period. This has reached the point that with the declining number of beds, it is the norm for the majority of in-patients on many National Health Service (NHS) psychiatric wards to be detained at any point in time. The reasons for this remain unclear, and are complicated by spatial variation. For example, London has consistently higher rates of compulsory in-patient treatment compared with the rest of England. ${ }^{5}$ Previous studies of psychiatric in-patient treatment have demonstrated higher rates among younger adults, ${ }^{6,7}$ and those of Black and minority ethnicity, ${ }^{8,9}$ as well as in urban environments and areas of social deprivation. ${ }^{10-13}$ However, a multilevel analysis of the rate of compulsory in-patient treatment identified that the majority of the variation in rates occurred at the individual level according to variables such as age and ethnicity. ${ }^{14}$ Area-level deprivation and ethnic density were also factors, but there was no independent effect of London once individual and area-level variables had been adjusted for. Our aims were therefore to record the rate of psychiatric in-patient treatment (voluntary and compulsory) in England in 2010/11 and describe the variation between rural and urban settings; to investigate whether the variation in these rates of in-patient treatment in rural and urban settings correlated with variations in levels of deprivation, ethnic density and age; and we hypothesised that differences in age,

†'See editorial, pp. 97-98, this issue. ethnic density and deprivation between urban and rural areas would explain differing rates of in-patient treatment.

\section{Method}

\section{Design}

This was an ecological study based on secondary analysis of routinely collected national data. The proportion of adults who spent time as a psychiatric in-patient during a 1-year period was the outcome measure. Information on in-patient treatment was gathered from the Mental Health Minimum Dataset (MHMDS) for the year 2010/11. Data from the MHMDS was linked to corresponding demographic data from the Office for National Statistics ${ }^{15}$ enabling rates to be calculated, and other variables to be measured including population age structure, ethnic density and levels of deprivation.

The year studied was 2010/11. The population data used was the mid-2011 population estimates from the Office for National Statistics based on the results of the 2011 census. The 2010 Index of Multiple Deprivation (IMD) median score for each primary care trust (PCT) was used as a measure of arealevel deprivation (sourced from http://www.communities.gov.uk/ publications/corporate/statistics/indices2010).

The setting was PCTs (geographically defined areas with a mean population size of 350000 , in which primary and secondary care NHS services were organised) in England. These were the smallest areas for which both denominator population data and in-patient data were available. Each PCT was categorised according to its urban or rural location. The rural/urban local authority 
classification (https://www.gov.uk/government/statistics/2001-ruralurban-definition-la-classification-and-other-geographies) was modified for the purpose of this study to make seven categories: inner London; outer London; other major urban: large urban (population $250000-750000$ ); small urban ( $<26 \%$ rural population); rural 2 (26-79\% rural population); rural 1 ( $+80 \%$ rural population).

Participants had all spent at least one day in a psychiatric hospital during the year 2010/11. Each individual was included only once irrespective of whether they had one or multiple admissions, or had been admitted prior to the year of study. Each participant was allocated to one of two groups according to the level of restriction they were subject to. The first was the voluntary in-patient treatment group consisting of patients who had been in a psychiatric hospital on a voluntary basis or who were detained for an initial assessment only $(72 \mathrm{~h}$ duration and in practice usually less than $24 \mathrm{~h}$ ). The compulsory in-patient treatment group consisted of any patient who had been detained in hospital at any point during the year for a longer period of assessment and/ or treatment. These patients could also have spent some time in hospital as voluntary patients as well during the year. It included civil detentions from the community (including from accident and emergency, or via the police, or other healthcare settings) and forensic detentions from a court of law or prison. The voluntary and compulsory treatment groups were mutually exclusive.

The MHMDS data were available for 143 out of the 152 PCTs in England. Five of these 143 PCTs were excluded as they received the majority of their mental health services from two mental health trusts that did not return data on compulsory treatment for the year of the study. Thus, data are presented on 138 PCTs. The 14 PCTs that were not included were from different areas of the country including both rural and urban settings.

\section{Statistical analysis}

For each PCT the adult population (aged 16 years plus), and the percentage aged 20 to 39 years of age were recorded. Rates of psychiatric in-patient treatment (overall, voluntary and compulsory) were calculated per 100000 of the adult population for each PCT. Average rates were calculated for the whole of England, and for each of the seven rural and urban settings. Spearman's rank correlations were calculated between average rates of treatment in each of the seven rural/urban settings and the corresponding rates of young adults and ethnic density, and deprivation scores in PCTs in these settings. Variations in the rates of compulsory in-patient treatment and the associations with other variables were then investigated in more detail. All statistical analyses were carried out using SPSS version 19.

\section{Results}

\section{Rates of psychiatric in-patient treatment}

The rate of in-patient psychiatric treatment was 276 (95\% CI 262-289) per 100000 adult population in the year 2010/11. This consisted of a rate of 159 (95\% CI 149-168) per 100000 of voluntary in-patient treatment and 117 (95\% CI 107-127) per 100000 of compulsory in-patient treatment.

\section{Rates in urban and rural locations}

Urban areas had higher rates of in-patient treatment, and the larger the urban environment the greater the rate of compulsory in-patient treatment, with rates highest in inner London (Table 1). Rates of voluntary treatment showed a different pattern with the highest rates seen in urban areas outside London, and less overall variation between rural and urban settings. Overall rates of in-patient treatment in inner London were $72 \%$ higher when compared with the most rural PCTs. Compulsory treatment rates were $184 \%$ higher but the rate of voluntary in-patient treatment was only $9 \%$ higher in inner London.

Age, ethnicity and deprivation also varied between these rural and urban categories. There was a strong or moderate association between levels of deprivation in these urban and rural settings and the rate of in-patient treatment for the corresponding area, both voluntary and compulsory. In addition there were strong associations between the percentage of the adult population aged 20-39 years and ethnic density with the rate of compulsory in-patient treatment, but no association with the rate of voluntary in-patient treatment (Table 2).

\section{Age and compulsory psychiatric in-patient treatment}

There was a nearly perfect correlation (Table 2) between the proportion of adults that were in their 20s and 30s and the rate of compulsory in-patient treatment. In more urban settings the proportion of adults aged 20-39 years steadily rose as did the rate of compulsory in-patient treatment. The only exception was a slight drop in the proportion of young adults between the large urban and major urban categories. However, there was a similar drop in the rate of compulsory in-patient treatment between these categories (Fig. 1).

\section{Area-level deprivation, ethnic density and compulsory psychiatric in-patient treatment}

The association between area-level deprivation and the rate of compulsory in-patient treatment was evident in both rural and urban areas (see Fig. 2). In contrast to deprivation, the association between compulsory in-patient treatment and ethnic density at the PCT level was only evident in urban settings. Figure 3 shows that rural PCTs had lower rates of ethnicity, and no association (or slightly negative association) between rates of compulsory in-patient treatment and ethnicity. Urban PCTs had much higher rates of ethnic density with a positive association between ethnicity and compulsory in-patient treatment.

In urban environments, rather than rural areas, the association between age and compulsory in-patient treatment is sustained after including interaction terms. The higher rates in urban areas $(P=0.06)$ increase further with a higher proportions of young adults $(P=0.05)$. No statistically significant interactions were found between urban areas and ethnicity $(P=0.12)$ or between urban areas and deprivation $(P=0.81)$.

\section{Variation in the rate of in-patient treatment}

In most areas average rates of voluntary in-patient treatment were $50-100 \%$ higher than rates of compulsory in-patient treatment. This applied to PCTs with below average rates of young adults where the average voluntary rate of in-patient treatment was $162(95 \%$ CI 150-173) per 100000 compared with a rate of 92 (95\% CI 84-100) per 100000 for compulsory in-patient treatment. PCTs with high rates of young adults tended towards similar rates (voluntary rate 154 (95\% 139-170), compulsory rate 154 (95\% 137-171), $n=55)$. Furthermore, in the 43 PCTs with both high proportions of young adults and high levels of ethnic density, average rates of compulsory in-patient treatment were higher $(167,95 \% 147-186)$ than rates of voluntary in-patient treatment (148, 95\% 132-165). This applied both in high and low deprivation areas. In total, $93 \%$ of PCTs in London had high proportions of young adults, compared with $46 \%$ of PCTs in other urban areas and $0 \%$ of PCTs in rural areas $\left(\chi^{2}=65.7\right.$, d.f. $\left.=2, P<0.001\right)$. 

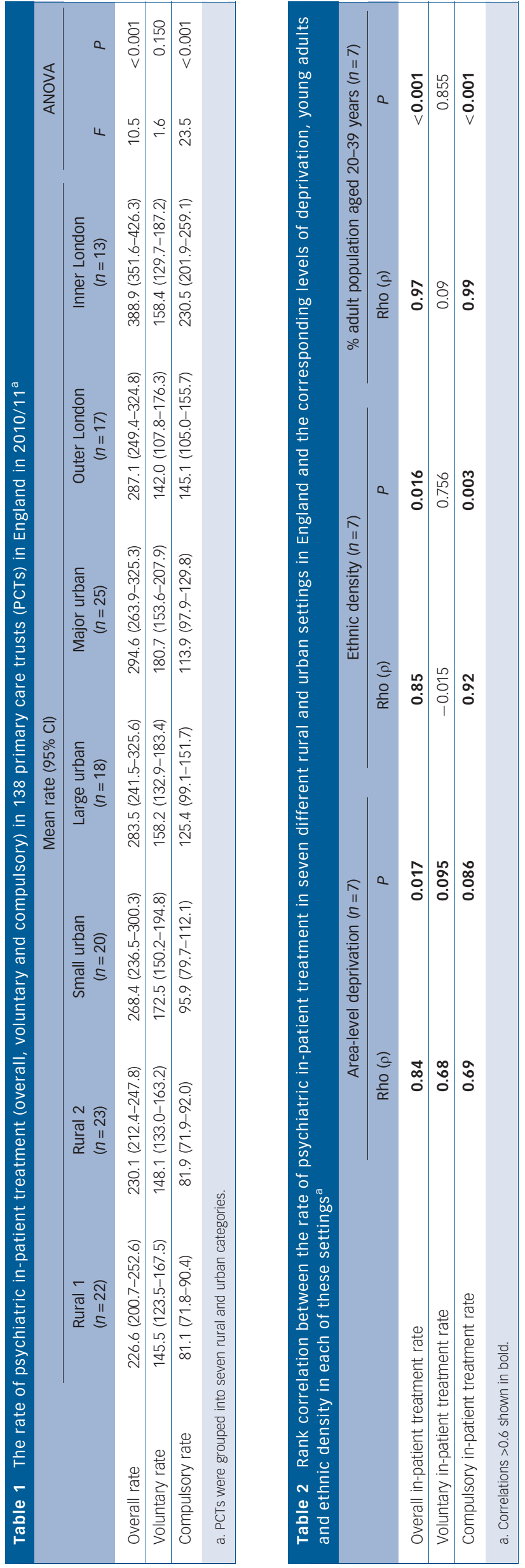

\section{Discussion}

\section{Main findings and implications}

This paper reports rates of voluntary and compulsory in-patient psychiatric treatment in seven rural and urban categories across England. The findings indicate that overall rates of in-patient treatment and compulsory rates increase in a stepwise fashion with urban environments: the larger the urban settings the greater the rate. A different pattern was seen for rates of voluntary in-patient treatment.

Our findings indicate that part of the explanation of the differences in rates between rural and urban areas is the age profile in these differing settings. Age, particularly young adulthood, came out as a strong explanatory variable in our multilevel analysis of the variation in rates of compulsory psychiatric admission. ${ }^{14}$ In the fully adjusted multilevel model the odds ratio for compulsory admission was 1.92 (95\% CI 1.82-2.02) in those aged 18-35, and 1.79 (95\% CI 1.68-1.89) in those aged 36-65 compared with those aged under 18.

We found an association between age and urban environments. Furthermore, our findings suggest that London may not have been identified as an explanatory variable in our original multivariate, multilevel models because of residual confounding by age and ethnicity. PCTs with above average proportions of adults in their 20 s and 30s had rates of compulsory in-patient treatment that were $67 \%$ higher and these PCTs were highly clustered in London. This important finding has implications for future research and service provision. We need to better understand which age-related variables lead to compulsory treatment. Service-related factors such as limited engagement with primary care and current mental health service structures may be important as well as factors that increase risk such as higher rates of impulsivity, suicide and violence. Any future comparisons of the use of compulsory treatment by mental health services will need to control for the age of the local population.

It is well established that rural areas in particular have low proportions of young adults. ${ }^{16}$ London had particularly high proportions of young adults, and in contrast to other areas had higher rates of compulsory treatment than voluntary treatment. There may be a number of possible explanations for why rates of voluntary admission were not higher in the most urban environments: pathways into care in an urban setting may be more likely to result in involuntary treatment; fewer older adults who are less likely to be detained live in these areas; the high rate of involuntary admission may limit the capacity for voluntary admission.

Deprivation was associated with rates of in-patient psychiatric treatment - both voluntary and compulsory. In contrast ethnicity was only associated with rates of compulsory in-patient treatment. It was also only in large and major urban areas, and particularly in London, that higher than average rates of deprivation, young adults and ethnic density were found together, and these areas had the highest rates of compulsory in-patient treatment.

As seen in other conditions ${ }^{17}$ there may be underlying contextual factors in these urban areas that invoke interactions between individuals and multiple vulnerabilities, leading to poorer health indices in general, and higher rates of compulsory treatment. Furthermore, the contextual factors in rural areas are likely to be different from urban areas. Our findings suggest that for a meaningful comparison to be made of rates of compulsory treatment between different mental health services, controlling for the setting in which each of the services operates will be vital. 


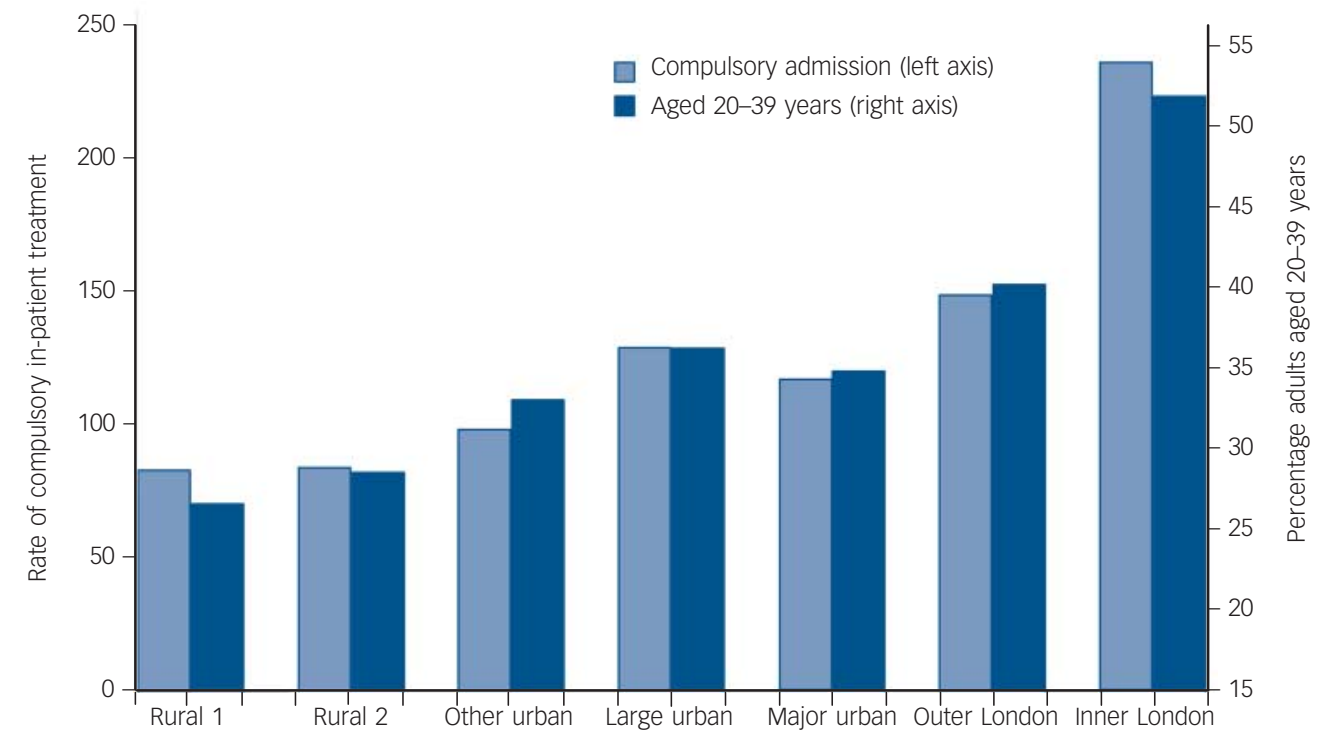

Fig. 1 The rate of compulsory in-patient treatment in rural and urban settings in England and the corresponding percentage of the adult population aged 20-39 years.

Data are for 138 primary care trusts in England in 2010/11.

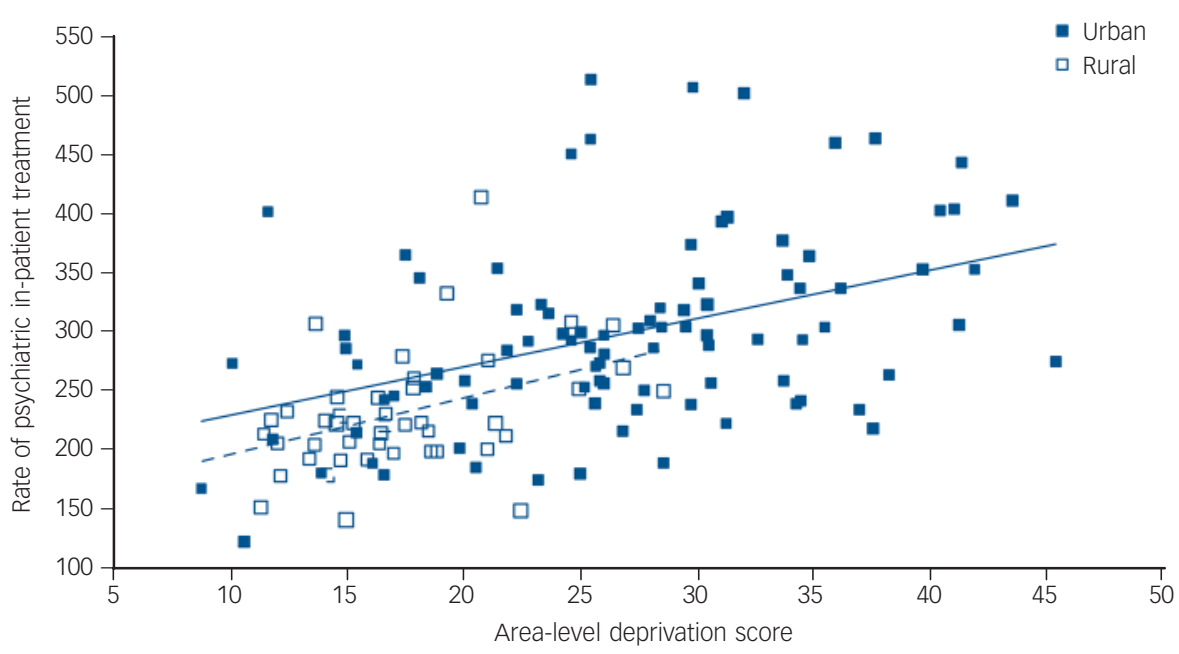

Fig. 2 Area-level deprivation in 45 rural primary care trusts (PCTS) and 93 urban PCTs in England and the corresponding rate of in-patient psychiatric treatment.

The solid line is the linear trend in urban areas and the dashed line is the linear trend in rual areas.

\section{Limitations}

Limitations of the study include that it is a secondary analysis of routinely collected data. In addition it is an ecological study and explores association at the population and group level rather than at the individual level. So associations can only be used to inform service delivery, and not individual-level interventions.

\section{Interpretation of our findings}

The age profile of each ethnic group in England varies considerably with the most minority ethnic groups being much younger that the majority White British population. ${ }^{18}$ Furthermore, many ethnic groups are highly clustered in major urban areas including London. Black and Asians groups in particular have been identified as being at greater risk of compulsory in-patient treatment. These groups make up just $1.3 \%$ of the rural population compared with $12.6 \%$ of the urban population, and
$20.7 \%$ of the population in major conurbations. ${ }^{19}$ Intriguingly, there is a suggestion in our results that rural areas with relatively high levels of ethnicity had some of the lowest rates of compulsory admission. This suggests that part of the explanation for the overrepresentation of ethnic groups among compulsory psychiatric in-patients may be that these groups tend to be younger and highly concentrated in urban areas. It may also explain why some of the ethnic groups with the lowest rates of compulsory in-patient treatment are more evenly spread through the country.

The population of England has risen steadily in the past 30 years and continues to grow. This growth has been largely confined to urban environments, and this may be part of the explanation for the increasing rates of compulsory admission that have taken place during this time period. ${ }^{20}$ There is a need to understand the distribution of compulsory admission in other countries with different jurisdictions to see if they show a similar pattern of concentration in large and major urban areas. 


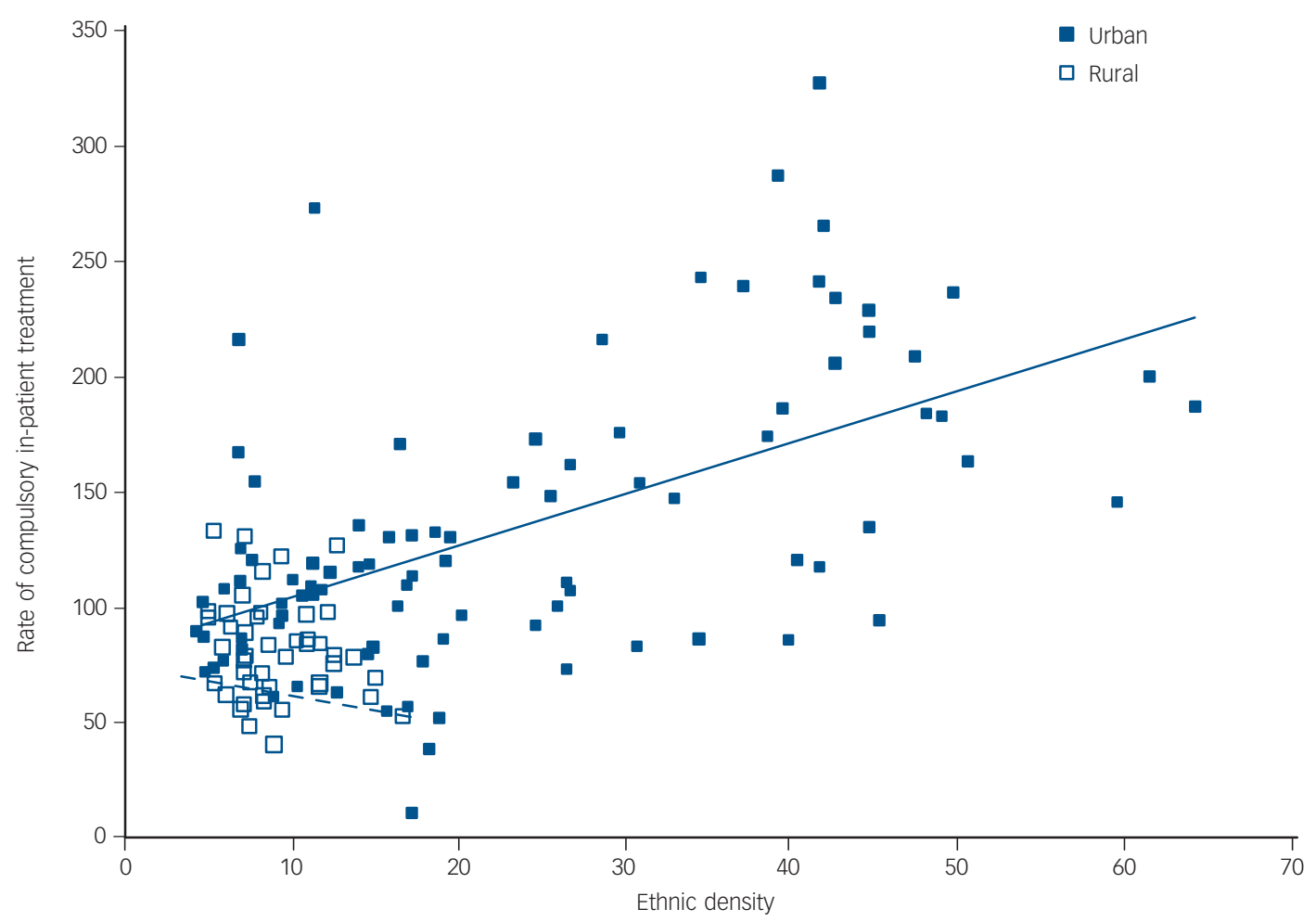

Fig. 3 The rate of compulsory in-patient treatment and ethnic density in 138 primary care trusts in England.

Data are shown separately for rural and urban areas. The solid line is the linear trend in urban areas and the dashed line is the linear trend in rual areas.

Patrick Keown, MRCPsych, Academic Psychiatry, Campus for Ageing \& Vitality, Newcastle University, Newcastle upon Tyne; Orla McBride, PhD, School of Psychology, University of Ulster, County Londonderry, Ulster; Liz Twigg, PhD, Department of Geography, University of Portsmouth, Portsmouth; David Crepaz-Keay, DProf, Mental Health Foundation, London; Eva Cyhlarova, DPhil, Department of Physiology, Anatomy and Genetics, University of Oxford, Oxford; Helen Parsons, PhD, Division of Health Sciences, Warwick Medical School, University of Warwick, Coventry; Jan Scott, MD, Academic Psychiatry, Campus for Ageing \& Vitality, Newcastle University, Newcastle upon Tyne; Kamaldeep Bhui, MD, Centre for Psychiatry, Barts and The London School of Medicine \& Dentistry, Queen Mary University of London, London; Scott Weich, MD, Division of Mental Health and Wellbeing, Warwick Medical School, University of Warwick, Coventry, UK

Correspondence: Patrick Keown, Northumberland, Tyne and Wear NHS Trust \& Newcastle University, Tranwell Unit, Queen Elizabeth Hospital, Gateshead NE10 9RW, UK. Email: patrick.keown@newcastle.ac.uk

First received 22 Jun 2015, final revision 14 Dec 2015, accepted 29 Dec 2015

\section{References}

1 Dean C, Phillips J, Gadd E, Joseph M, England S. Comparison of a community based service with a hospital based service for people with acute, severe psychiatric illness. BMJ 1993; 307: 473-6.

2 Johnson S, Nolan F, Pilling S, Sandor A, Hoult J, McKenzie N, et al. Randomised controlled trial of acute mental health care by a crisis resolution team: the north Islington crisis study. BMJ 2005; 331: 586-90.

3 Craig TKJ, Garety $\mathrm{P}$, Power $\mathrm{P}$, Rahaman $\mathrm{N}$, Colbert S, Fornells-Ambrojo $\mathrm{M}$, et al. The Lambeth Early Onset (LEO) Team: randomised controlled trial of the effectiveness of specialised care for early psychosis. BMJ 2004; 329: 1067-71.

4 Glover G, Arts G, Babu KS. Crisis resolution/home treatment teams and psychiatric admission rates in England. Br J Psychiatry 2006; 189: 441-5.

5 The Information Centre. Inpatients Formally Detained in Hospitals under the Mental Health Act 1983 and Other Legislation. Government Statistical Service, 2011.

6 Thompson A, Shaw M, Harrison G, Ho D, Gunnell D, Verne J. Patterns of hospital admission for adult psychiatric illness in England: analysis of Hospital Episode Statistics data. Br J Psychiatry 2004; 185: 334-41.
7 Audini B, Lelliott P. Age, gender and ethnicity of those detained under Part II of the Mental Health Act 1983. Br J Psychiatry 2002; 180: 222-6.

8 Bhui K, Stansfield S, Hull S, Priebe S, Mole F, Feder G. Ethnic variations in pathways to and use of specialist mental health services in the UK. Br J Psychiatry 2003; 182: 105-16.

9 Singh SP, Greenwood N, White S, Churchill R. Ethnicity and the Mental Health Act 1983. Br J Psychiatry 2007; 191: 99-105.

10 Faris REL, Dunham HW. Mental Disorders in Urban Areas: An Ecological Study of Schizophrenia and Other Psychoses. Hafner, 1939.

11 Ineichen B, Harrison G, Morgan HG. Psychiatric hospital admissions in Bristol. I. Geographical and ethnic factors. Br J Psychiatry 1984; 145: 600-4.

12 Kirkbride JB, Errazuriz A, Croudace TJ, Morgan C, Jackson D, Boydell J, et al. Incidence of schizophrenia and other psychoses in England, 1950-2009: a systematic review and meta-analyses. PLOS One 2012; 7: e31660.

13 Van der Linden J, Gunther N, Feron F, van Os J. Children's mental health service use, neighbourhood socioeconomic deprivation, and social capital. Soc Psychiatry Psychiatr Epidemiol 2003; 38: 507-14.

14 weich S, Mcbride O, Twigg L, Keown P, Cyhlarova E, Crepaz-Keay D, et al. Variation in compulsory psychiatric inpatient admission in England: a crosssectional, multilevel analysis. Health Serv Deliv Res 2014; 2: 90.

15 Office for National Statistics. Primary Care Organisations Mid-Year Population Estimates, Mid 2011 (Census Based). Office for National Statistics, 2013 (http://www.ons.gov.uk/ons/rel/sape/pco-pop-est-exp/mid-2011-final-censusbased-/stb—primary-care-organisation—mid-2011-census-based-.html).

16 Pateman T. Rural and urban areas: comparing lives using rural/urban classifications. Regional Trends 2011; 43: 62-3.

17 Falzon D. The city, its people, their health and tuberculosis. Eurosurveillance 2014; 19 (9): 2-3.

18 Office for National Statistics. Detailed Characteristics for England and Wales, March 2011. Office for National Statistics, 2013 (http://www.ons.gov.uk/ons/ dcp171778_310514.pdf).

19 National Online Manpower Information System. KS201EW - Ethnic Group. National Online Manpower Information System, 2011 (https:// www.nomisweb.co.uk/census/2011/ks201ew).

20 Keown P, Weich S, Bhui K, Scott J. Association between the provision of mental illness beds and rate of involuntary admissions in the NHS in England: ecological study 1988-2008. BMJ 2011; 343: d3736. 